\title{
СИНЕРГЕТИКА ИНФОРМАЦИОННО-КОГНИТИВНОГО ВЗАИМОДЕЙСТВИЯ В ИНТЕАИЕКТУААЬНЫХ РОБОТОТЕХНИЧЕСКИХ СИСТЕМАХ С ДИСТАНЦИОННЫМ ОБМЕНОМ ЗНАНИЯМИ
}

\author{
С.В. Ульянов, д.ф.-м.н., профессор; \\ А.Г. Решетников, к.т.н., доиент, agreshetnikov@gmail.com \\ (Университет "Дубна", Институт системного анализа и управления, \\ ул. Университетская, 19, г. Дубна, 141980, Россия)
}

\begin{abstract}
В статье представлена технология дистанционного проектирования БЗ нечетких регуляторов, разработанных с использованием программного инструментария «Оптимизатор Баз Знаний» на мягких и квантовых вычислениях. Рассматривается возможность настройки и передачи БЗ с использованием дистанционного подключения к объекту управ-
\end{abstract} ления.

Предложенные технологии позволяют повысить робастность системы управления за счет добавления функций обучения и адаптации к различным ситуациям управления. Представлено сравнение качества управления в нечетких регуляторах при работе в различных режимах. Настройка и передача БЗ нечеткого регулятора реализуются при помощи удаленного соединения с объектом управления в online с использованием технологий Bluetooth и Wi-Fi. B качестве примеров выступают различные модели интеллектуальных автономных роботов.

Дистанционная передача БЗ позволяет проектировать множество различных встраиваемых интеллектуальных регуляторов для реализации множества стратегий управления в условиях неопределенности и риска. Реализованная технология обмена знаниями в группе интеллектуальных роботов позволяет гарантированно достигать цели управления и извлекать дополнительные знания за счет формирования нового информационного источника, основанного на синергетическом эффекте объединения знаний.

В статье рассматриваются различные варианты взаимодействия робототехнических систем. Приведено краткое описание каждой из систем. Результаты экспериментов демонстрируют возможность гарантированного достижения цели управления коллективом роботов за счет использования технологий мягких вычислений при проектировании БЗ нечетких регуляторов.

Разработанный программный инструментарий дает возможность проектировать и настраивать сложные и слабо формализованные технические системы. Данная возможность позволяет существенно сократить время при проектировании интеллектуальной системы управления и повысить надежность системы за счет снижения уровня влияния экспертных оценок на процесс проектирования.

Ключевые слова: коллектив интеллектуальных роботов, многоагентная система, интеллектуальное управление, нечеткий регулятор, дистанционная передача знаний, синергетика знаний.

Одной из ключевых задач современной робототехники является развитие технологий когнитивного взаимодействия робототехнических систем, позволяющих решать задачи интеллектуального иерархического управления за счет перераспределения знаний и функций управления, например, традиционно между лидером и подчиненным (master-slave system). Современные подходы к решению данной задачи основаны на теории многоагентных систем, теории роевого искусственного интеллекта и многих других [1-8].

В многоагентной системе существует, согласно [9-14], новый синергетический информационный эффект самоорганизации БЗ и формирования дополнительного информационного ресурса, возникающий при обмене информацией и знаниями между активными агентами (swarm synergetic information effect) $[9,10]$. Извлечение дополнительного ресурса в виде скрытой в классических состояниях квантовой информации осуществляется на основе квантового нечеткого вывода, который, в свою очередь, является новым квантовым поисковым алгоритмом и частным случаем квантового алгоритма самоорганизации.
За счет синергетического эффекта создается дополнительный информационный ресурс, и многоагентная система способна решать сложные динамические задачи по выполнению совместной работы. Поставленная задача может не выполняться каждым элементом (агентом) системы в отдельности в разнообразных средах без внешнего управления, контроля или координации, но обмен знаниями и информацией позволяет совершать совместную полезную работу для достижения поставленной цели управления в условиях неопределенности исходной информации и ограничений на расход полезного ресурса [10]. В частности, известно, что для систем управления с обратной связью количество извлекаемой полезной работы $W$ удовлетворяет неравенству $W_{\max }(t)=k \int_{0}^{t} T_{\min } \dot{I}_{c} d t^{\prime} \leq$ $\leq k T I_{c}$, где $k$ - постоянная Больцмана; $T_{\min }(t)$ интерпретируется как наименьшая достижимая системой температура во времени $t$ при управлении с обратной связью в предположении, что $T_{\min }(0)=T$, и $I_{c}$ определяет количество информации Шеннона (перенос энтропии), извлекаемое системой из процесса измерения $[13,14]$. 
Физически синергетический эффект - это самоорганизация знаний и создание дополнительной информации, позволяющей многоагентной системе совершать максимально полезную работу при минимуме потерь полезного ресурса и требуемой исходной информации, без разрушения нижнего исполнительного уровня системы управления $[11,12,14]$. Совместно с информационно-термодинамическим законом интеллектуального управления (оптимальное распределение качеств «устойчивость-управляемость-робастность») проектируется интеллектуальная система управления (ИСУ) многоагентными системами, гарантирующая достижение цели управления в условиях неопределенности исходной информации и ограниченного полезного ресурса $[10-12,14]$.

Речь идет о сети слабо связанных между собой роботов, совместно решающих задачи, которые выходят за рамки индивидуальных возможностей. Различные узлы подобной системы, как правило, имеют различный уровень интеллектуализации (знания, алгоритмы, вычислительные базисы) и разные информационные ресурсы при проектировании. Каждый узел должен быть способен модифицировать свое поведение в зависимости от обстоятельств, а также планировать свои стратегии коммуникации и кооперации с другими узлами. Здесь показателями уровня кооперации являются характер распределения задач, объединение различных информационных ресурсов и, конечно, возможность решения общей проблемы в заданное время $[4,5]$.

\section{Актуальность проблемы и постановка задачи}

В условиях неопределенности или неточности исходной информации, непредвиденных ситуаций или информационного риска традиционный (использующий принцип глобальной отрицательной обратной связи) и широко применяемый в промышленности ПИД-регулятор часто не справляется с поставленной задачей управления. В то же время решение задачи о глобальной робастности ПИД-регулятора до настоящего времени неизвестно, несмотря на актуальность данной проблемы.

Использование нечетких регуляторов (НР) совместно с ПИД-регулятором привело к созданию гибридных нечетких ИСУ с различными уровнями интеллектуальности в зависимости от полноты и корректности спроектированной БЗ. Использование технологии мягких вычислений (основанной на генетических алгоритмах (ГА) и нечетких нейронных сетях (ННС)) расширило области эффективного применения НР за счет добавления новых функций в виде обучения и адаптации.

Разработанный интеллектуальный инструментарий Оптимизатор баз знаний (ОБ3) SCOptKB ${ }^{\mathrm{TM}}$ позволил проектировать робастные БЗ на основе решения одной из алгоритмически трудно решаемых задач теории искусственного интеллекта - извлечение, обработка и формирование объективных знаний без использования экспертных оценок [12]. В данном ОБЗ применяются три ГА, которые позволяют спроектировать оптимальную структуру НР (вид и число ФП, их параметры, а также число самих правил нечеткого вывода), аппроксимирующего обучающий сигнал с требуемой ошибкой. При этом автоматически проектируется оптимальная структура ННС и формируется модель универсального аппроксиматора в виде НР с конечным числом продукционных правил в БЗ.

Технологии дистанционной настройки и передачи БЗ позволяют объекту управления (ОУ) принимать БЗ от блока ОБЗ или от других ОУ, что дает возможность управлять структурно новыми объектами, такими как коллективы роботов, многоагентные системы, сложные автоматизированные производства и др. Кроме того, данная технология дает возможность ОУ обновлять и адаптировать БЗ под конкретную ситуацию управления, в том числе нештатную.

Интенсификация производственного процесса, рост сложности и количества различных технических изделий, повышение вычислительной мощности процессоров свидетельствуют о необходимости развития технологий проектирования и внедрения интеллектуальных систем в контур управления. В свою очередь, необходимо наличие интеллектуального инструментария для настройки подобных систем в различных предметных областях. Повышение вычислительного базиса (типов вычислений) программного продукта приводит к повышению качества и надежности управления, добавляя свойства адаптации и обучения в контур обратной связи. Однако повышение надежности системы управления ведет к увеличению настраиваемых параметров и, как следствие, к повышению сложности настройки подобных систем.

Разработанный инструментарий реализует механизмы создания, настройки и передачи параметров управления в виде сигналов управления, полученных из БЗ НР, не разрушая нижний исполнительный уровень. Использование мягких вычислений и разработанных технологий проектирования ИСУ сокращает влияние экспертных оценок в процессе обучения и настройки ИСУ.

Для демонстрации взаимодействия робототехнических систем были спроектированы макеты роботов. Системы управления роботов функционируют на основе нейросетевых НР, разработанных на основе технологии, представленной в $[9,10]$.

\section{Структура и основные функции ОБЗ}

Применительно к проектированию ИСУ мягкие вычисления представляют собой комбинацию из 


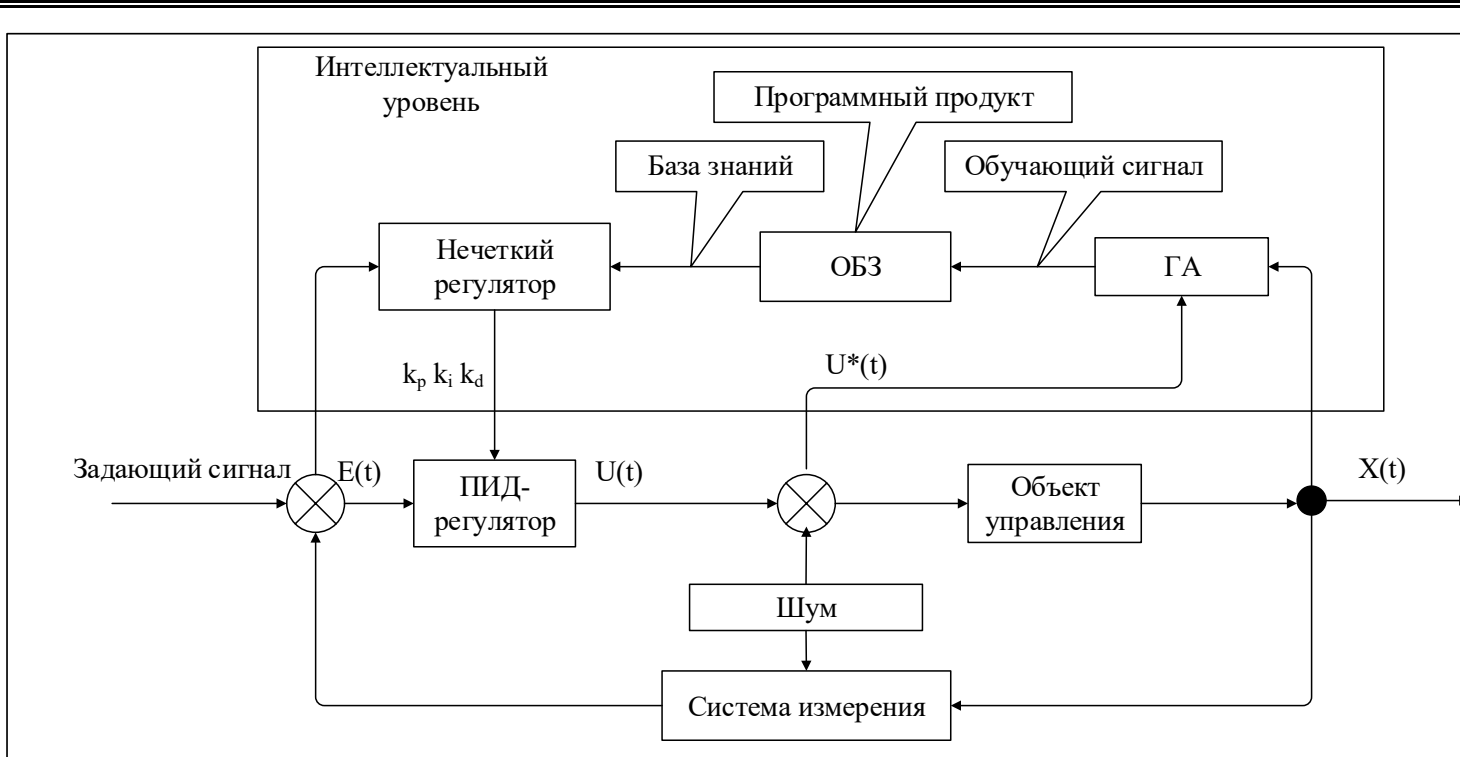

Рис. 1. Структура ИСУ с НР и ОБЗ

Fig. 1. The structure of an intelligent control system with a fuzzy controller and a knowledge base optimizer

следующих подходов: теория нечетких систем, ГА и ННС. НР является центральным элементом ИСУ и вырабатывает управляющие сигналы изменения во времени (законы управления) коэффициентами усиления $k_{p}, k_{d}, k_{i}$ (coefficient gain schedule) ПИДрегулятора.

Функциональная структура ИСУ с блоками НР и ОБЗ на мягких вычислениях представлена на рисунке 1. Такая структура включает одновременно следующие качества управления: управляемость, точность и устойчивость (нижний уровень управления - САУ), обучение и адаптация (верхний интеллектуальный уровень управления - НР с БЗ).

Входом ОБЗ является обучающий сигнал, который может быть получен либо на этапе стохастического моделирования поведения ОУ (с использованием его математической модели), либо экспериментально, то есть непосредственно из результатов измерений динамических параметров физической модели ОУ.

ОС является источником знаний и представляет собой массив данных, разделенный на входные и выходные составляющие, каждая из которых, в свою очередь, состоит из одного и более сигналов. В случае аппроксимации некоторого управляющего сигнала входными компонентами могут являться ошибка управления, интеграл ошибки и ее производная, а выходным компонентом - требуемое значение управляющего воздействия либо некоторые настраиваемые параметры системы управления, например, коэффициенты усиления ПИД-регулятора. Результатом аппроксимации обучающего сигнала является построенная Б3 для НР, включающая оптимальное конечное множество правил и оптимально сформированные параметры функции принадлежности входных и выходных переменных НР.
Таким образом, результатом проектирования является требуемый тип универсального аппроксиматора в виде НР с оптимальной структурой БЗ.

\section{Дистанционное соединение с ОБ3 и передача Б3}

Дистанционная настройка позволяет проектировать ИСУ, получая данные непосредственно с ОУ без использования системы стохастического моделирования, что дает преимущество при проектировании нечетких систем управления для сложных и слабо формализованных ОУ в непредвиденных ситуациях управления.

Рассмотрим модуль удаленного соединения ОБ3 и реального ОУ для настройки БЗ. Для передачи данных используется USB-соединение или радиоканал Bluetooth. Происходит обмен информацией между системой управления и ОБЗ для формирования Б3 (рис. 2).

Система управления считывает показания датчиков и отправляет их на компьютер для последующей обработки. Приняв входные значения, ОБ3 оценивает в функции ГА предыдущее решение (Б3 НР) и осуществляет нечеткий вывод для проверки следующего решения (Б3 НР). Результат нечеткого вывода отправляется на удаленное устройство. После этого система управления, обработав входные значения, вырабатывает управляющее воздействие. Таким образом, реализуется настройка Б3 HP в online. Профиль соединения использует последовательный порт. Скорость передачи в нашем случае составляет 115200 бит/с. В процессе функционирования по СОМ-порту передаются числа в символьном виде. Подключение к ОБЗ осуществляется через разработанный плагин. 


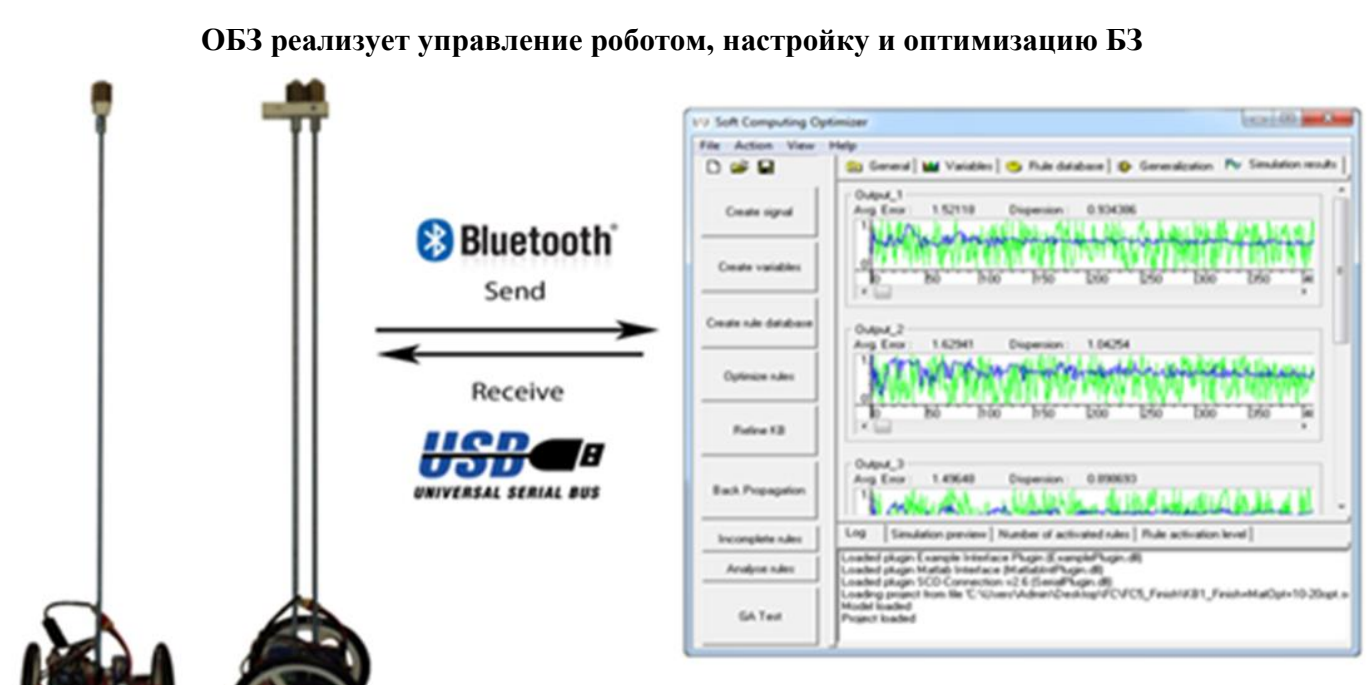

Робот передает данные с сенсоров и управляет исполнительными механизмами

Рис. 2. Схема соединения настраиваемого устройства и ОБЗ

Fig. 2. The scheme of connection between an adjustable device and a knowledge base optimizer

Удаленная передача Б3 является следующим шагом развития беспроводного соединения ОУ с ОБЗ. В данном случае от ОБЗ передаются не управляющие воздействия, а Б3, то есть происходит обмен информацией и знаниями более высокого уровня. Реализация среды соединения предполагает использовать стандарт IEEE 802.11 (Wi-Fi) и протокол ТCP/IP для приема-передачи данных. Осуществляется обмен информацией между системой управления и ОБЗ для формирования и передачи БЗ (рис. 3).

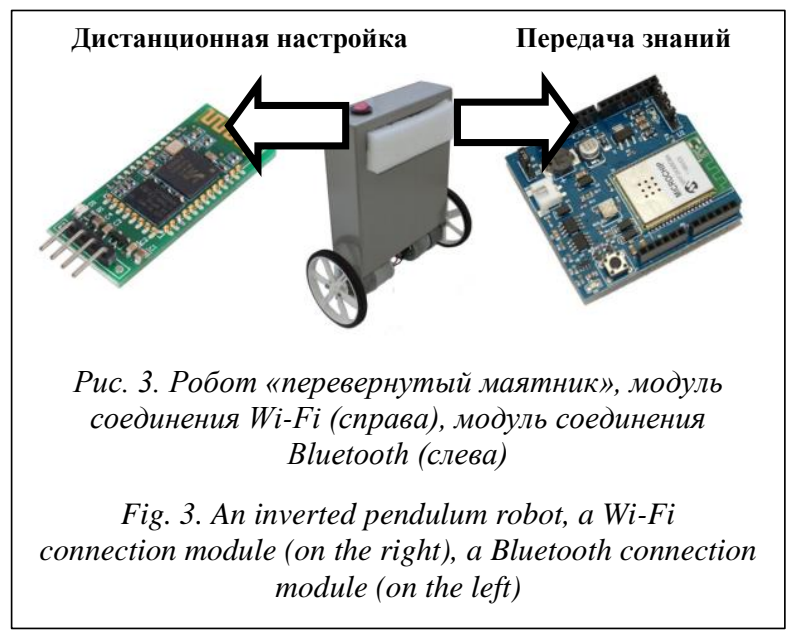

Структурно БЗ с точки зрения программной инженерии реализована структурным типом данных, и ее размер зависит от количества входных и выходных переменных, количества функций принадлежности лингвистических переменных и числа продукционных правил. При скорости 1 Мбит/с передача БЗ размером 10 Кбайт с учетом внутренних задержек составляет не больше 100 мс, что позво- ляет качественно перестраивать ИСУ под заданную задачу в режиме online.

Проведем сравнение ПИД-регулятора (PID) с $\mathrm{HP}$ (FC1), оптимизированного с помощью дистанционной настройки. На рисунке 4а представлен угол отклонения сравниваемых регуляторов, а на

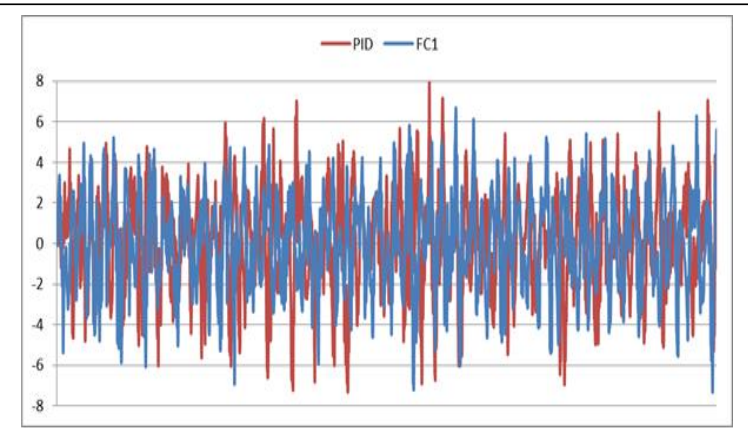

a)

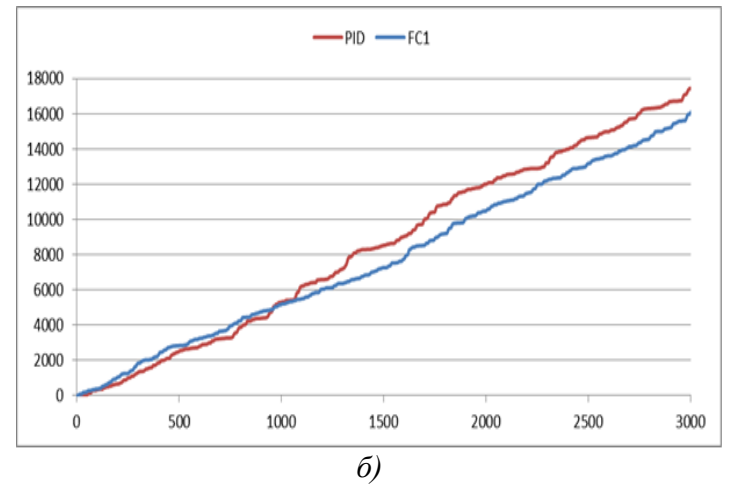

Рис. 4. Угол отклонения перевернутого маятника (а), интегральная оценка угла отклонения (б)

Fig. 4. A deflection angle of an inverted pendulum (a), an integral estimation of a deflection angle ( $\sigma)$ 
рисунке 4б - интегральная оценка угла отклонения в типовой ситуации. На рисунке 5а представлен уровень отъезда левого (left) и правого (right) колес от точки запуска, а на рисунке 56 - интегральная оценка отъезда.

Как показывают рисунки 4 и 5, уровень ошибки НР ниже ПИД-регулятора и для угла отклонения, и для отъезда от точки запуска.

\section{Моделирование и эксперимент взаимодействия группы роботов}

В качестве ОУ выступают макеты манипулятора, перевернутого маятника и мобильного манипулятора. Мобильный и стационарный манипуляторы оснащены системой распознавания образов на основе библиотеки компьютерного зрения OpenCV и аппаратной части в виде web-камеры, приставки Kinnect и инфракрасного сенсора. Рассматриваются децентрализованные и иерархические варианты взаимодействия группы роботов. Демонстрируется эксперимент в связке ведущийведомый (master-slave), проводимый на основе системы распознавания мобильного манипулятора.

В качестве демонстрационных роботов используются перевернутый маятник, тележка с манипулятором и стационарный робот-манипулятор.

Перевернутый маятник представляет собой динамически неустойчивый мобильный объект. Разработанная ИСУ позволяет роботу свободно перемещаться по различным видам поверхностей, таким как паркет, асфальт, линолеум, стол, а также дополнительно ставить на него объекты различной тяжести, например бокал, телефон и т.п. На рисунке 6а представлена трехмерная модель перевернутого маятника.

Тележка с манипулятором представляет собой мобильную платформу с установленным на нее манипулятором с тремя степенями свободы. Для ориентации в пространстве на робот установлены две

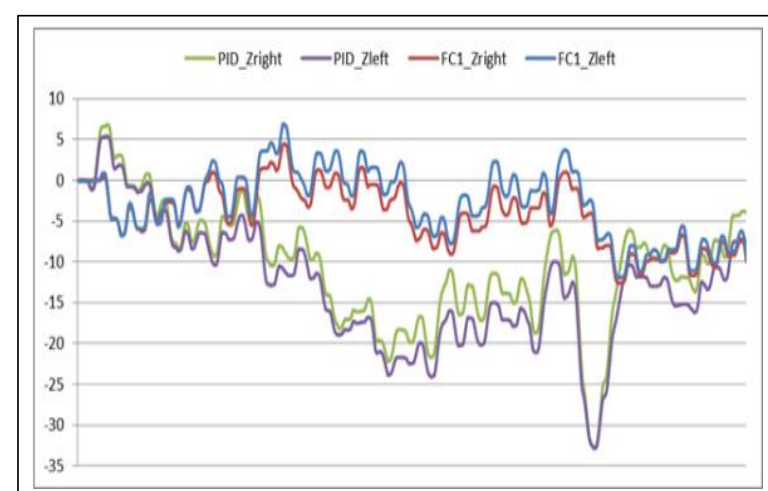

a)

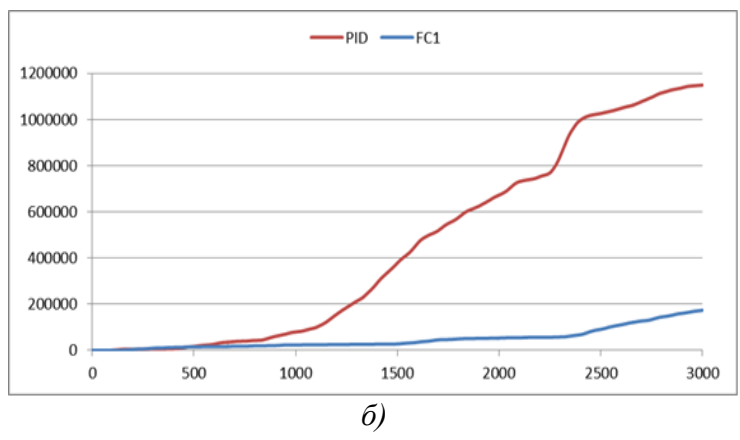

Рис. 5. Отъезд правого и левого колес от точки запуска (а), интегральная оченка отъезда от точки запуска (б)

Fig. 5. A pullback of the right and left wheels from the start point (a), integrated assessment of a pullback from the start point

камеры: цветная Kinect и инфракрасная Wiimote. Для распознавания объектов взаимодействия робот использует систему распознавания образов, работающую в среде OpenCV. Система может свободно перемещаться в пространстве, распознавать различные объекты и использовать манипулятор для взаимодействия с ними. Модель тележки представлена на рисунке $6 б$.

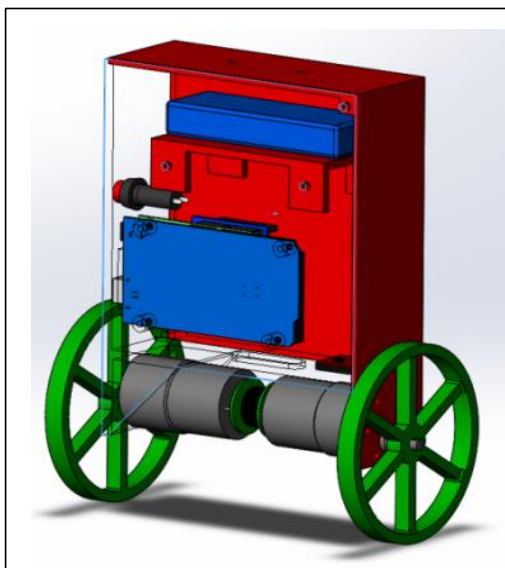

a)

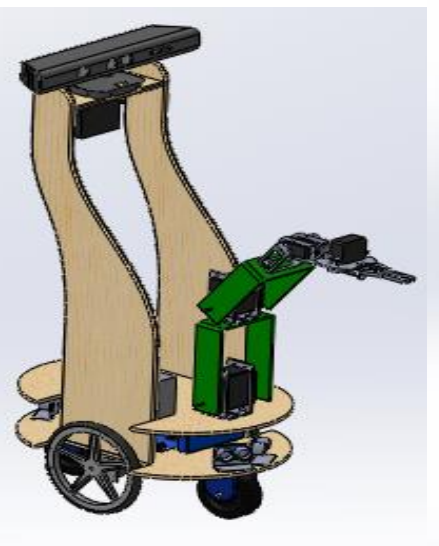

б)

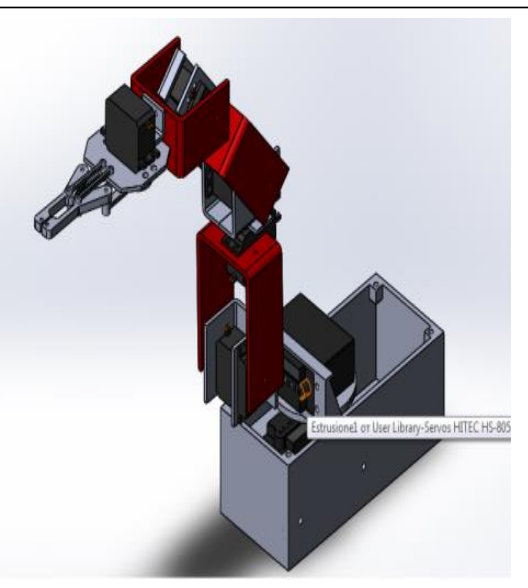

в)

Рис. 6. Трехмерная модель: а) перевернутого маятника, б) тележки с манипулятором, в) робота-манипулятора

Fig. 6. A three-dimensional model of: a) an inverted pendulum, б) a manipulator truck, в) a robot manipulator 

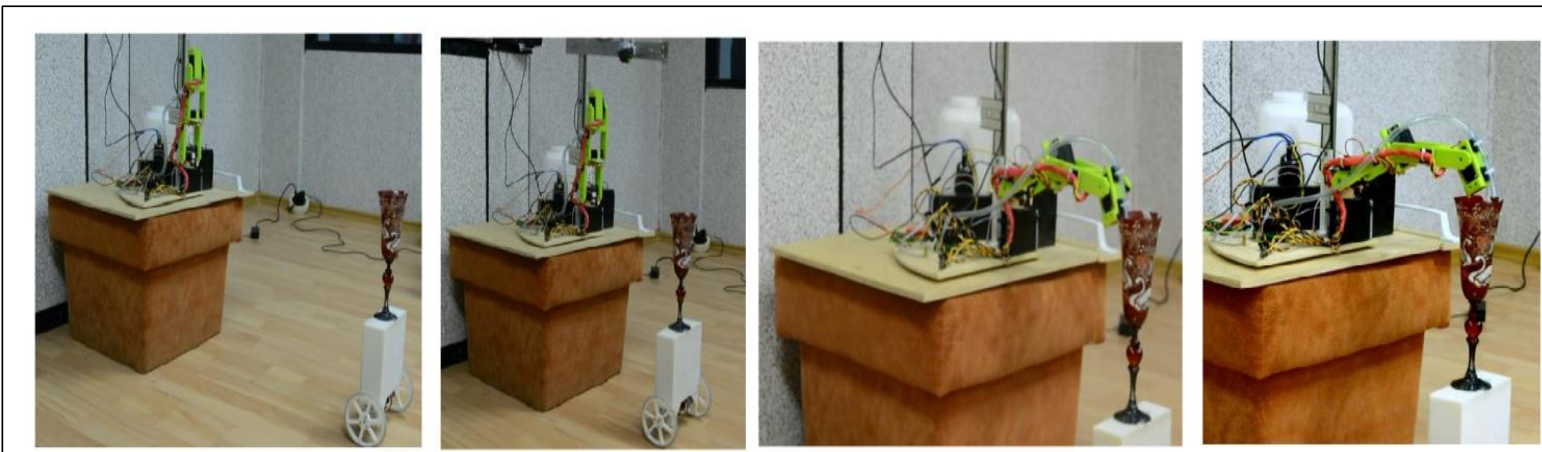

Рис. 7. Робот-манипулятор наливает жидкость в бокал, установленный на перевернутом маятнике

Fig. 7. The robot manipulator pours the liquid into the glass installed on inverted pendulum

Стационарный робот-манипулятор представляет собой манипулятор с четырьмя степенями свободы. Робот оснащен камерой, охватывающей обзором поле рядом с манипулятором и системой распознавания контуров. Вместо захвата к манипулятору подведена трубка с жидкостью, которая хранится в бачке. Робот способен распознавать контуры емкостей, размещенных в рабочей области, и наполнять их какой-либо жидкостью. На рисунке 6в представлена трехмерная модель стационарного манипулятора.

Рассмотрим реализованные типы взаимодействия в группе роботов. В первом демонстрационном примере на робот типа перевернутый маятник [15-17] устанавливается бокал, который является объектом распознавания для стационарного манипулятора. Перевернутый маятник определяет свое положение относительно робота-манипулятора с помощью инфракрасной камеры и «маячков», при соответствующей команде меняет свое местоположение и перемещается к манипулятору в требуемую точку пространства.

Когда перевернутый маятник оказывается в зоне видимости стационарного манипулятора, система распознавания определяет местоположение бокала и отправляет манипулятору команду наполнить его жидкостью (рис. 7).

Такое взаимодействие является типовым, и его применение возможно в целом спектре задач. Кроме очевидных возможностей автоматизации кафе и баров, возможна автоматизация множества производственных задач, таких как погрузка-разгрузка контейнеров, сортировка и т.п.

В качестве второго примера взаимодействия рассматривается задача, в которой сверху на перевернутый маятник устанавливается бокал. Тележка с манипулятором оснащена инфракрасной камерой, определяющей относительное положение перевернутого маятника, и начинает движение в сторону распознанного объекта. Также команда на сближение отправляется перевернутому маятнику. После сближения мобильный манипулятор с использованием системы распознавания и камеры Kinect определяет местоположение бокала. Ма- нипулятор, оснащенной ИСУ, рассчитывает команду двигателям в звеньях и перемещает захват манипулятора к бокалу. Сначала тележка находит в зоне видимости перевернутый маятник и определяет его положение. Если маятник повернут, ему отправляется команда на разворот. Если маятник стоит ровно, начинается взаимное движение навстречу. Когда дистанция становится оптимальной для захвата бокала, в работу включается камера Kinect и происходит распознавание бокала, установленного на маятник. После распознавания бокала отправляются координаты и команда манипулятору на захват бокала (рис. 8).

Такое взаимодействие реализует иерархическое управление в связке ведущий и ведомый. Так, перевернутый маятник выступает в роли ведомого и выполняет команды от мобильного манипулятора, который обладает дополнительным сенсором для определения положения маятника.

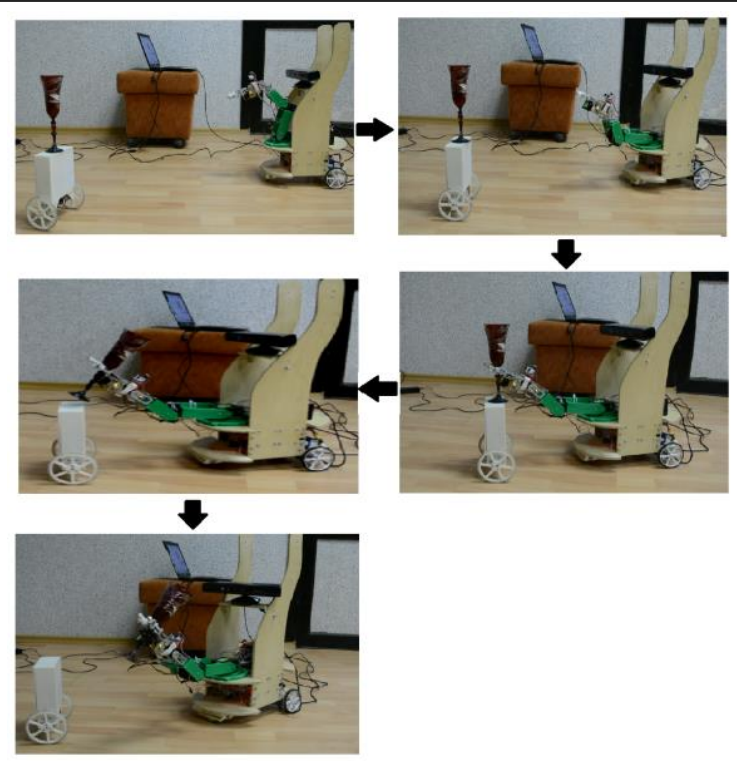

Рис. 8. Мобильный манипулятор выполняет захват бокала с перевернутого маятника

Fig. 8. The mobile manipulator performs a capture of the glass from an inverted pendulum 


\section{Выводы}

Результаты проведенных исследований показали следующее:

- удаленная оптимизация БЗ вместе с ГАПИД-регулятором позволяют строить ИСУ без использования математического моделирования в режиме online;

- ОБЗ обеспечивает повышение надежности ИСУ в режиме online;

- дистанционное соединение позволяет оптимизировать Б3 в определенных (типовых) условиях функционирования, тем самым создавая множество Б3 с высоким качеством управления для различных ситуаций управления;

- инструментарий позволяет настраивать сложные системы управления с большим количеством контуров управления; количество входных переменных и размер получаемой Б3 ограничиваются лишь аппаратными характеристиками компьютера и ОУ.

Разработанные варианты взаимодействия показывают возможности применения данных систем в широком спектре задач, таких как автоматизация складов и производств, автоматические заведения общественного питания, ликвидация последствий чрезвычайных происшествий и т.п. Главной особенностью многоагентной системы является синергетический эффект, возникающий от объединения нескольких робототехнических систем, когда результат функционального взаимодействия намного превышает возможности каждого из роботов в отдельности.

\section{Литература}

1. Тарасов В.Б. От многоагентных систем к интеллектуальным организациям: философия, психология, информатика. М.: Эдиториал УРСС, 2002. 352 с.

2. Зайцев А.А., Курейчик В.В., Полупанов А.А. Обзор эволюционных методов оптимизации на основе роевого интеллекта // Изв. ЮФУ: Технические науки. 2010. № 12. С. 7-12.
3. Cazenille L., Bredeche N., Halloy J. Automated optimization of multi-level modes of collective behavior in a mixed society of animals and robots. URL: https://arxiv.org/pdf/1602.05830v1.pdf (дата обращения: 2.02.2017).

4. Dudek G., Jenkin M., Milios E., Wilkes D. A taxonomy for multi-agent robotics. Autonomous Robots, 1996, vol. 3, iss. 4, pp. 375-397.

5. Ota J. Multi-agent robot systems as distributed autonomous systems. Advanced Engineering Informatics, 2006, vol. 20, iss. 1, pp. 59-70.

6. Kawamura K., Gordon S. From Intelligent Control to Cognitive Control. Proc. World Automation Congress (WAC '06), 2006, pp. $1-8$.

7. Kawamura K., Peters R.A., Bodenheimer R., et all. Multiagent-based cognitive robot architecture and its realization. Intern. Jour. of Humanoid Robotics, 2004, vol. 1, no. 1, pp. 65-93.

8. Lenk J.C., Droste R., Sobiech C., Ludtke A., Hahn A. Towards cooperative cognitive models in multi-agent systems. Proc. Intern. Conf. on Advanced Cognitive Technologies and Applications, 2012, pp. 67-70.

9. Ulyanov S.V., et all. Quantum swarm model of self-organization process on quantum fuzzy inference and robust wise control design. Proc. $7^{\text {th }}$ Intern. Conf. Appl. of Fuzzy Systems and Soft Computing (ICAFS'2006), Siegen, Germany, 2006, pp. 10-19.

10. Ulyanov S.V. Self-organizing quantum robust control methods and systems for situations with uncertainty and risk. Patent US 8788450 B2, 2014

11. Ульянов С.В., Решетников Г.П., Решетников А.Г. Технологии интеллектуальных вычислений. Квантовые вычисления и программирование в самоорганизующихся интеллектуальных системах управления. Дубна: Изд-во ОИЯИ, 2015.

12. Ульянов С.В., Николаева А.В., Решетников А.Г. Интеллектуальные системы управления в непредвиденных ситуациях. Оптимизатор баз знаний на мягких вычислениях. LAP LAMBERT Acad. Publ., OmniScriptum GmbH \& Co. KG, 2013.

13. Sandberg $H$., et all. Maximum work extraction and implementation costs for nonequilibrium Maxwell's demon. Physical Review E, 2014, no. 4, pp. 042-119.

14. Ульянов С.В. Квантовая релятивистская информатика. LAP LAMBERT Acad. Publ., OmniScriptum GmbH \& Co. KG, 2015.

15. Nawawi S.W., Ahmad M.N., and Osman J.H.S. Real-time control system for a two-wheeled inverted pendulum mobile - robot. Advanced Knowledge Application in Practice, InTech, 2010, pp. 299-312.

16. Gocmen A. Design of two wheeled electric vehicle. Master Sci. Thesis. Atilim Univ., Temmuz, 2011.

17. Castro A. Modeling and dynamic analysis of a twowheeled inverted pendulum. Master Sc. Thesis, Georgia Institute of Technology, Atlanta, USA, 2012.

\section{SYNERGETICS OF INFORMATION-COGNITIVE INTERACTION IN INTELLIGENT ROBOTIC SYSTEMS WITH REMOTE KNOWLEDGE EXCHANGE}

S.V. Ulyanov ${ }^{1}$, Dr.Sc. (Physics and Mathematics), Professor

A.G. Reshetnikov ${ }^{1}$, Ph.D. (Engineering), Associate Professor, agreshetnikov@gmail.com

${ }^{1}$ Dubna University - Institute of System Analysis and Control, Universitetskaya St. 19, Dubna, 141980, Russian Federation

Abstract. The article describes a technology of knowledge bases remote design for fuzzy controllers, which are developed using the software toolkit "Knowledge base optimizer" based on soft and quantum computing. The paper also considers the 
possibility of tuning and transferring a knowledge base using remote connection to a controlled object. The presented technologies allow increasing control system robustness by adding training and adapting functions to various management situations. There is a comparison of control quality in fuzzy controllers operating in various control modes. Configuring and transferring fuzzy controller knowledge bases is performed using a remote connection with a control object online via Bluetooth and WiFi. As examples, there are different models of intelligent autonomous robots. Remote transmission of knowledge bases allows designing many different built-in intelligent regulators to implement a variety of control strategies under uncertainty and risk. The implemented technology of knowledge sharing in the group of intelligent robots allows achieving the goal of control and gaining additional knowledge by creating a new information source based on the synergistic effect of combining knowledge.

The article considers various options of interaction between robotic systems. There is a brief description of each system. The experimental results demonstrate the possibility of guaranteed achievement of the control goal by a group of robots using soft computing technologies when designing knowledge bases of fuzzy controllers. The developed software toolkit allows designing and configuring complex ill-defined and poorly formalized technical systems online. This feature significantly reduces the time for intelligent control system design and improves system reliability by reducing the level of influence of expert estimates on the design process.

Keywords: group of intelligent robots, multi-agent system, intelligent control, fuzzy controller, remote transmission of knowledge, knowledge synergetic.

\section{References}

1. Tarasov V.B. Ot mnogoagentnykh sistem k intellektualnym organizatsiyam: filosofiya, psikhologiya, informatika [From Multi-Agent Systems to Intellectual Organizations: Philosophy, Psychology, Informatics]. Moscow, Editorial URSS Publ., 2002, 352 p.

2. Zaytsev A.A., Kureychik V.V., Polupanov A.A. Evolution methods of optimization research based on swarm intelligence. Izvestiya YuFU. Tekhnicheskie nauki [Izvestiya SFedU. Engineering Sciences]. 2010, no. 12, pp. 7-12 (in Russ.).

3. Cazenille L., Bredeche N., Halloy J. Automated optimization of multi-level modes of collective behavior in a mixed society of animals and robots. Available at: https://arxiv.org/pdf/1602.05830v1.pdf (accessed February 2, 2017).

4. Dudek G., Jenkin M., Milios E., Wilkes D. A taxonomy for multi-agent robotics. Autonomous Robots. 1996, vol. 3, iss. 4, pp. 375-397.

5. Ota J. Multi-agent robot systems as distributed autonomous systems. Advanced Engineering Informatics. 2006, vol. 20 , iss. 1, pp. 59-70.

6. Kawamura K., Gordon S. From intelligent control to cognitive control. Proc. World Automation Congr. WAC '06. 2006, pp. $1-8$.

7. Kawamura K., Peters R.A., Bodenheimer R., Sarkar N., Spratley A., Hambuchen K.A. Multiagent-based cognitive robot architecture and its realization. Int. Jour. of Humanoid Robotics. 2004, iss. 1 (1), pp. 65-93.

8. Lenk J.C., Droste R., Sobiech C., Ludtke A., Hahn A. Towards cooperative cognitive models in multi-agent systems. Proc. Int. Conf. on advanced cognitive technologies and applications. 2012, pp. 67-70.

9. Ulyanov S.V., Litvintseva L.V., Ulyanov S.S. Quantum swarm model of self-organization process on quantum fuzzy inference and robust wise control design. Proc. 7th Int. Conf. on Appl. of Fuzzy Systems and Soft Computing (ICAFS'2006). Siegen, Germany, 2006, pp. 10-19.

10. Ulyanov S.V. Self-Organizing Quantum Robust Control Methods and Systems for Situations with Uncertainty and Risk. Patent US 8788450 B2, 2014

11. Ulyanov S.V., Reshetnikov G.P., Reshetnikov A.G. Tekhnologii intellektualnykh vychisleny. Kvantovye vychisleniya $i$ programmirovanie $v$ samoorganizuyushchikhsya intellektualnykh sistemakh upravleniya [Quantum Computing and Programming in Self-Organizing Intelligent Control Systems]. Dubna, JINR, 2015.

12. Ulyanov S.V., Nikolaeva A.V., Reshetnikov A.G. Intellektualnye sistemy upravleniya v nepredvidennykh situatsiyakh [Intelligent Control Systems in Unforeseen Situations]. LAP Lambert Academic Publ., OmniScriptum GmbH \& Co. KG, 2013.

13. Sandberg H., Delvenne J.C., Newton N.J., Mitter S.K. Maximum work extraction and implementation costs for nonequilibrium Maxwell's demon. Physical Review E. 2014, vol. 90, no. 4, pp. 042-119.

14. Ulyanov S.V. Kvantovaya relyativistskaya informatika [Quantum relativistic informatics]. LAP LAMBERT Academic Publ., OmniScriptum GmbH \& Co. KG, 2015.

15. Nawawi S.W., Ahmad M.N., Osman J.H.S. Real-time control system for a two-wheeled inverted pendulum mobile robot. Advanced Knowledge Application in Practice. InTech Publ., 2010, pp. 299-312.

16. Gocmen A. Design of two-wheeled electric vehicle. Master Sci. Thesis. Atilim Univ., 2011.

17. Castro A. Modeling and dynamic analysis of a two-wheeled inverted pendulum. Master Sci. Thesis, Georgia Institute of Technology, Atlanta, USA, 2012. 